\title{
KATANIN 1 Is Essential for Embryogenesis and Seed Formation in Arabidopsis
}

\author{
Ivan Luptovčiak ${ }^{+}$, Despina Samakovlit, George Komis and Jozef Šamaj *
}

Centre of the Region Haná for Biotechnological and Agricultural Research, Faculty of Science, Palacký University Olomouc, Olomouc, Czechia

Cytoskeletal remodeling has a fundamental role, especially during transitional developmental stages when cells rapidly adopt new forms and roles, like gametogenesis, fertilization and concomitant embryogenesis and seed formation. KATANIN 1, a microtubule severing protein, fulfills a major regulatory mechanism of dynamic microtubule turnover in eukaryotes. Herein, we show that three well-established KATANIN 1 mutants, fra2, lue1 and ktn1-2 collectively display lower fertility and seed set in Arabidopsis. These lower fertility and seed set rates of fra2, lue1 and ktn1-2 mutants were correlated to abnormalities in the development of embryo

OPEN ACCESS

Edited by:

Stefan de Folter

Center for Advanced Research The National Polytechnic Institute,

Mexico

Reviewed by:

Frantisek Baluska,

University of Bonn, Germany

Simona Masiero,

Università degli Studi di Milano, Italy

${ }^{*}$ Correspondence:

Jozef Šamaj

jozef.samaj@upol.cz

tThese authors have contributed equally to this work.

Specialty section:

This article was submitted to Plant Evolution and Development,

a section of the journal

Frontiers in Plant Science

Received: 12 December 2016 Accepted: 19 April 2017

Published: 05 May 2017

Citation:

Luptovčiak I, Samakovli D, Komis G and Šamaj J (2017) KATANIN 1 is

Essential for Embryogenesis and Seed Formation in Arabidopsis.

Front. Plant Sci. 8:728.

doi: 10.3389/fpls.2017.00728 proper and seed. Such phenotypes were rescued by transformation of mutants with functional pKTN1::GFP:KTN1 construct. This study significantly expands the already broad functional repertoire of KATANIN 1 and unravels its new role in embryo and seed development. Thus, KATANIN 1 significantly contributes to the fertility and proper embryo and seed formation in Arabidopsis.

Keywords: Arabidopsis, development, katanin, embryo, embryogeneis, seed, microtubule

\section{INTRODUCTION}

In angiosperms, seeds arise from a fertilized ovule and consist of the following parts: the embryo which arises from the zygote, the endosperm which arises from the fertilization of the diploid central cell by haploid sperm cell and the integuments. Cell division pattern pinpoints the development and growth of all three parts and for this reason is crucial for both embryonic and post-embryonic plant development (Jürgens and Mayer, 1994; ten Hove et al., 2015).

The regulation of microtubule dynamics is essential for numerous cellular functions. The ability to accurately control microtubule number, the rate of microtubule assembly and disassembly, and the assembly of microtubule networks governs cellular processes including differentiation, division and migration. A major regulatory mechanism of dynamic microtubule turnover in eukaryotes, involves microtubule severing by means of AAA-ATPase family proteins including katanin (McNally and Vale, 1993; Burk et al., 2001), fidgetin (Mukherjee et al., 2012) and spastin (Roll-Mecak and Vale, 2005). In plants as represented by the genetically tractable model Arabidopsis thaliana, only katanin has been identified as a microtubule severing protein (Burk et al., 2001; McClinton et al., 2001), and was shown thereon to play central roles in mechanisms governing microtubule organization. Typically, the mammalian AAA-ATPase katanin is assembled by a catalytic subunit of roughly $60 \mathrm{kDa}$ (p60) and a structural $80 \mathrm{kDa}$ subunit (p80; Hartman et al., 1998). For severing activity, katanin forms hexameric rings on the surface of microtubules and exerts its catalytic activity using ATP hydrolysis (Hartman and Vale, 1999). In the Arabidopsis genome, only the p60 subunit is expressed (designated as KATANIN 1; although four putative 

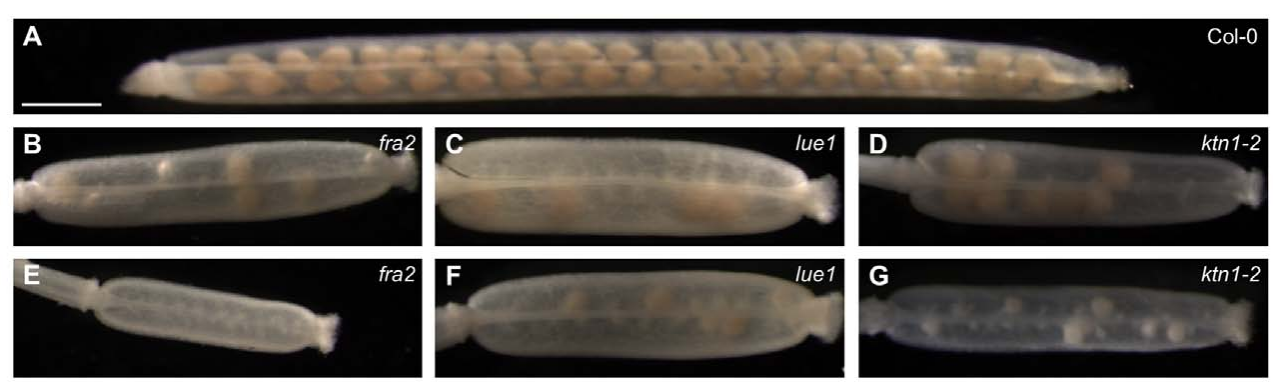

H

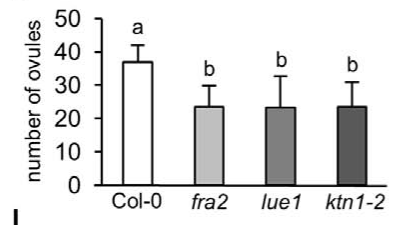

$$
\text { I }
$$

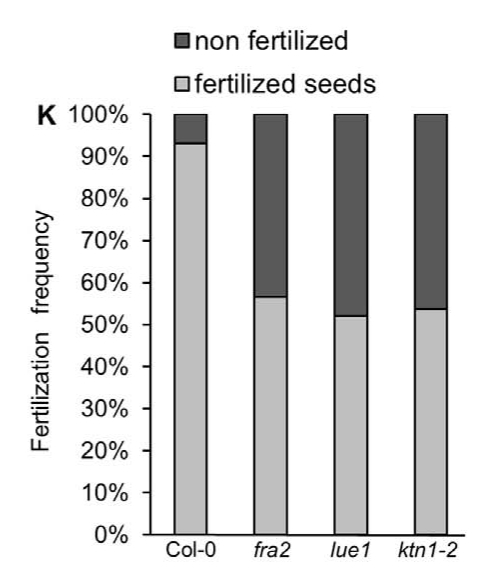

FIGURE 1 | Development of siliques and fertilized seeds in KATANIN 1 mutants. (A-G) Representative images of developing seeds in cleared siliques of Col-0 (A) and KATANIN 1 mutants: fra2 (B), lue1 (C), and ktn1-2 (D), and asynchronous seed development in fra2 (E), lue1 (F), and ktn1-2 (G). (H) Number of total ovules per silique in Col-0 and KATANIN 1 mutants. (I) Number of non-fertilized seeds per silique in Col-0 and KATANIN 1 mutants. (J) Number of developing seeds per silique in Col-0 and KATANIN 1 mutants. (K) The ratio of non-fertilized and fertilized seeds in siliques of Col-0 and KATANIN 1 mutants. Final calculations were based on data collection from 13 to 29 siliques. Different lowercase letters indicate statistical significance between treatments $(p<0.001)$. Error bars show \pm SD. Scale bar $=1 \mathrm{~mm}$.

p80 orthologs have been identified; Keech et al., 2010) and it is capable of severing microtubules in an ATP-dependent manner (Stoppin-Mellet et al., 2007). At the cellular level, the severing activity of KATANIN 1 was shown to regulate important aspects of plant microtubule organization (Nakamura, 2015). KATANIN 1 was shown to sever nascent, $\gamma$-tubulin-nucleated microtubules, growing from the walls of pre-existing microtubules (Nakamura et al., 2010) and also microtubules that are crossing each other during their dynamic excursions (Wightman and Turner, 2007; Soga et al., 2010; Lindeboom et al., 2013; Zhang et al., 2013) favoring in this way the biased parallel arrangement of cortical microtubules. Moreover, KATANIN 1 activity favors microtubule bundle formation (Stoppin-Mellet et al., 2006) and can be modulated by other microtubule binding proteins like SPIRAL2 (Wightman et al., 2013).

Previous studies have revealed that Arabidopsis KATANIN 1 mutants display pleiotropic phenotypes with defects affecting almost all vegetative organs (Bichet et al., 2001; Burk et al., 2001; Burk and Ye, 2002; Webb et al., 2002; Bouquin et al., 2003; Panteris et al., 2011; Panteris and Adamakis, 2012;
Lindeboom et al., 2013; Zhang et al., 2013; Abu-Abied et al., 2015a,b). However, the role of KATANIN 1 in Arabidopsis embryogenesis and seed set regulation has not been addressed so far. To gain insight to KATANIN 1 role we analyzed defects in embryogenesis and seed formation in well established fra2, lue1 and ktn1-2 mutants. Mutants fra2 and lue1 are rather similar as in both a truncated p60 is produced: in fra2 due to a deletion at nucleotide 2329 (Burk and Ye, 2002) and in lue 1 because of a single base change producing a non-sense mutation at amino acid 394 (Bouquin et al., 2003). Mutant ktn1-2 contains a single T-DNA insertion after the 147th nucleotide in the 5th exon of KATANIN 1 (Nakamura et al., 2010). In this study, we quantitatively surveyed several developmental aspects including embryogenesis and seed formation in above mentioned KATANIN 1 mutants. Phenotypical abnormalities in embryogenesis and seed formation of KATANIN 1 mutants were rescued by complementation of these mutants with pKTN1::GFP:KTN1 construct. Obtained results strongly supported a new role of KATANIN 1 in the embryogenesis and proper seed formation in Arabidopsis. 

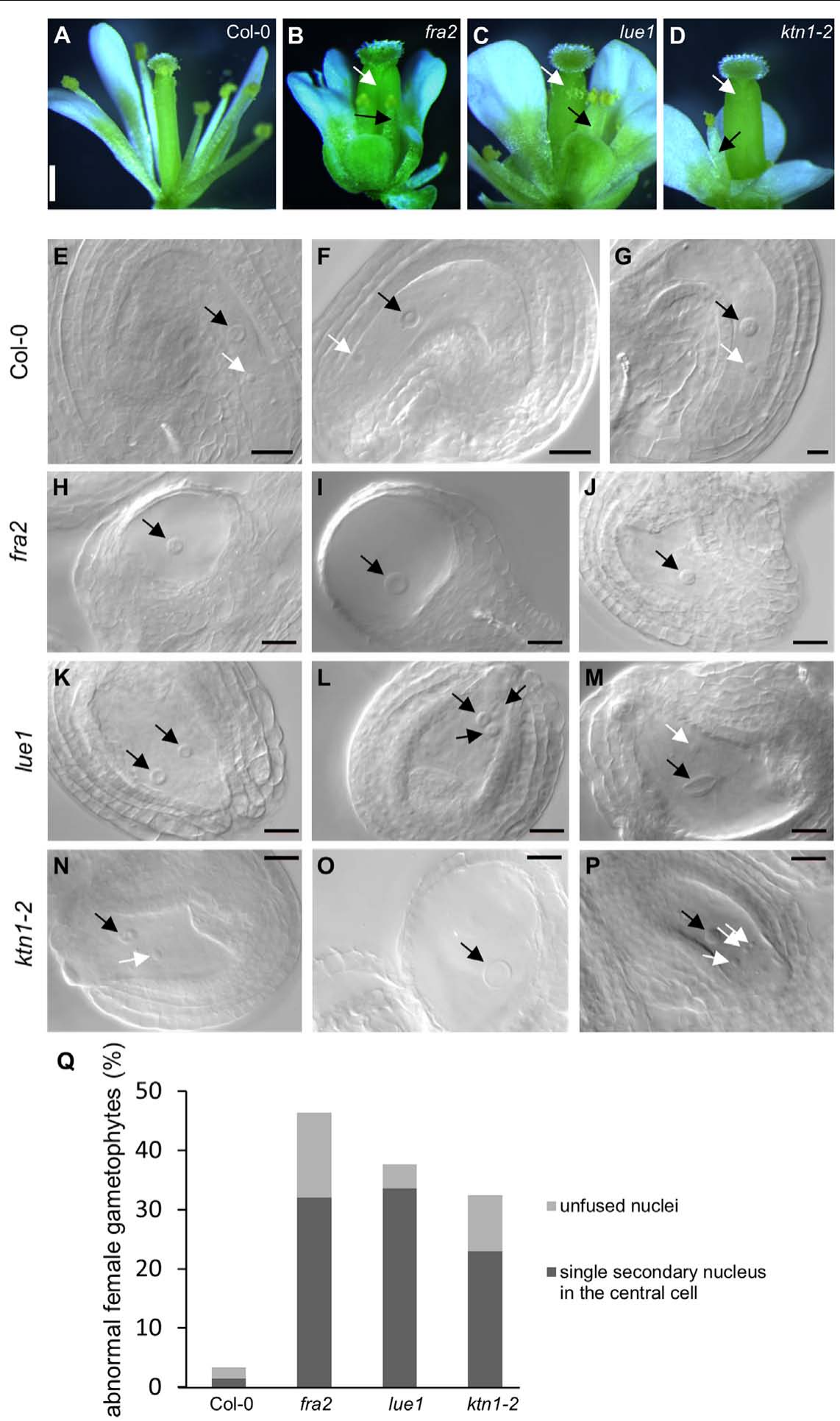

FIGURE 2 | Developmental defects in flowers and embryo sacs of KATANIN 1 mutants. (A-D) Representative pictures images of flowers of Col-0 (A) and KATANIN 1 mutants fra2 (B), lue1 (C), and ktn1-2 (D). Defective stamens and pistils are depicted with black and white arrows. (E-G) Wild type embryo sacs. The central cell nucleus (black arrows) and the egg cell nucleus (white arrows) were in proximal distance close to the micropylar pole showing that the migration and the precise positioning of the polar nuclei have been normally performed. (H-J) fra2 embryo sacs showed abnormalities in the migration and the positioning of nuclei and in the most cases only the central cell nucleus was present. Abnormalities in the development of the outer and inner integuments have been also observed (H,I) (K-M) lue1 embryo sacs. Misplacement of the polar nuclei (K) and unfused or double central cell nuclei (L,M). (N-P) ktn1-2 embryo sacs. Misplacement of the polar nuclei (N), unfused nuclei (P), highly vacuolated central cell nuclei, absence of egg cell nucleus $(\mathbf{O})$, abnormalities in the development of the outer and inner integuments (N-P). (Q) Quantification of the abnormal ovule phenotypes. Scale bars $=500 \mu \mathrm{m}$ (A-D); $50 \mu \mathrm{m}$ (E-P). 

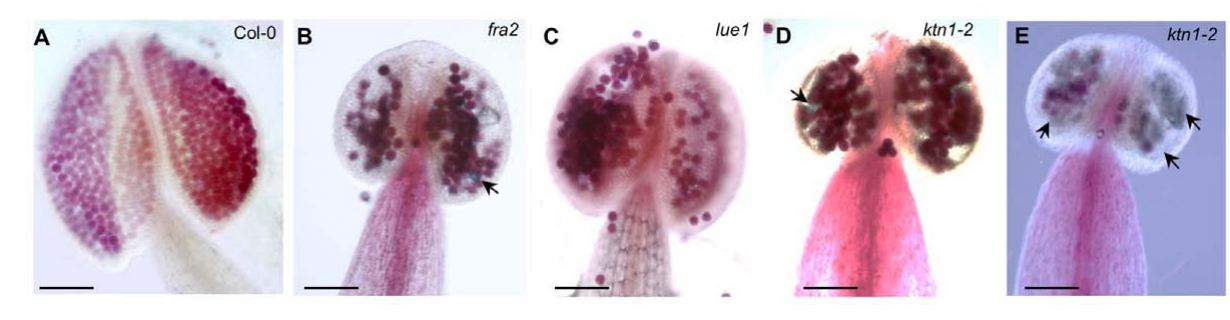

FIGURE 3 | Developmental defects in anthers of KATANIN 1 mutants. (A-E) Representative images of Alexander solution stained pollen in anthers of Col-0 (A) and KATANIN 1 mutants fra2 (B), lue1 (C), and ktn1-2 (D,E). Arrows point to the unviable shrunken pollen stained green by Alexander solution (B,D,E). Scale bars $=100 \mu \mathrm{m}(\mathbf{A}-\mathbf{E})$

\section{MATERIALS AND METHODS}

\section{Plant Material}

Arabidopsis thaliana wild type Columbia (Col-0) ecotype, fra2, lue 1 and $k t n 1-2$, T-DNA mutant were used. For germination, Col-0 and mutant seeds were surface sterilized, plated on $0.8 \%$ w/v Phytagel ${ }^{\circledast}$ solidified $1 / 2$ Murashige and Skoog medium $\left(1 / 2\right.$ MS) with $1 \% \mathrm{w} / \mathrm{v}$ sucrose, stratified for $1-4$ days at $4^{\circ} \mathrm{C}$ and subsequently transferred to environmental chamber with controlled light/dark cycle, temperature and humidity.

\section{Pollen Viability Analysis with Alexander Solution Staining}

For analysis of pollen viability, stamens from floral buds were placed on a microscopic slide. A few drops of Alexander stain buffer (95\% v/v ethanol, $10 \mathrm{~mL}$; Malachite green ( $1 \% \mathrm{w} / \mathrm{v}$ in $95 \%$ $\mathrm{v} / \mathrm{v}$ ethanol), $1 \mathrm{~mL}$; Fuchsin acid ( $1 \% \mathrm{w} / \mathrm{v}$ in water), $5 \mathrm{~mL}$; Orange $\mathrm{G}(1 \% \mathrm{w} / \mathrm{v}$ in water), $0.5 \mathrm{~mL}$; phenol, $5 \mathrm{~g}$; chloral hydrate, $5 \mathrm{~g}$; glacial acetic acid, $2 \mathrm{~mL}$; glycerol, $25 \mathrm{~mL}$; distilled water, $50 \mathrm{~mL}$ ) were added. Stained pollen grains were observed with a Zeiss Axio Imager A1 microscope equipped with differential contrast interference microscopy optics. After staining viable pollen is purple, while dead pollen is green.

\section{Embryo Development}

For embryo development, siliques at variable developmental stages were removed from Col-0, fra2, lue 1 and ktn1-2 plants, fixed in $50 \% \mathrm{v} / \mathrm{v}$ ethanol and $10 \% \mathrm{v} / \mathrm{v}$ acetic acid in water and cleared in chloral hydrate solution as described above. Following clearing, siliques were dissected in a drop of chloral hydrate solution on a glass slide to extract ovules. Samples were imaged and documented with DIC optics of a Zeiss AxioImager microscope equipped with a Zeiss MRc5 digital camera.

\section{Cloning of pKTN1::GFP:KTN1 Construct}

The N-terminal fusion construct of enhanced GFP (eGFP) with AtKTN1 driven under its own promoter ( $p K T N 1:: G F P: K T N 1$ ) was prepared using genomic DNA from leaf tissue. The $1143 \mathrm{bp}$ AtKTN1 (AT1G80350) promoter upstream of ATG start codon was amplified using respective primers: 5'-GGGGACAACT TTGTATAGAAAAGTTGTGCCTGCAGATAGCTTACTCAG-3' and 5'-GGGGACTGCTTTTTTGTACAAACTTGGCCTCTTTT ACTAAAAAAATAGCC-3'.

AtKTN1 genomic sequence for $\mathrm{N}$-terminal fusion (GFP is fused with N-terminus of KTN1) was amplified using primers: 5'-GGGGACAGCTTTCTTGTACAAAGTGGGCATGGTGGGA AGTAGTAATTCGTTAGCC-3' and 5'-GGGGACAACTTTGTA TAATAAAGTTGCTTAAGCAGATCCAAACTCAGAGAG-3'.

Amplified promoter, GFP sequence (plasmid pEN-L1-F-L2 MultiSiteGateway ${ }^{\circledR}$ ) and AtKTN1 genomic DNA were assembled using recombination reaction according to MultiSite Gateway ${ }^{\circledR}$ Three-Fragment Vector Construction Kit and cloned into pB7m34GW.0, which was then used for Agrobacterium tumefaciens GW3101 transformation. Col-0 and KATANIN 1 mutants were transformed with this construct using established floral dip method.

\section{RESULTS}

\section{Abberant Fertility of KATANIN 1 Mutants}

All KATANIN 1 mutants showed reduced fertility, which is evident by the formation of numerous non-elongating siliques on the inflorescences of fra2, lue 1 and ktn1-2, suggesting that irregular development of siliques arised from defects in fertilization (Supplemental Figures 1A-D). In all three mutants, fra2, lue1 and ktn1-2, mature siliques were shorter compared to Col-0 (Figures 1A-G and Supplemental Figures 1A-H). Col-0 siliques showed synchronous seed development (Figure 1A), but in fra2, lue 1 and ktn1-2 the number of ovules per silique was roughly half (Figures 1A-H). More detailed studies showed unfertilized ovules (Figures 1I,K) containing only few developing seeds (Figures 1B-G,J,K).

\section{Defects in Ovule Development in KATANIN 1 Mutants}

In mature flowers of KATANIN 1 mutants the pistils display developmental defects, such as reduced size and abnormal carpel junction (Figures 2B-D), while the stamens grow separately from the female organs showing decreased filament length unable to reach the pistils for successful pollination (Figures 2A-D), as previously described ( $\mathrm{Qu}$ et al., 2012). There were some differences among the KATANIN 1 mutants in the flower patterning and the development of the four whorls constituting 

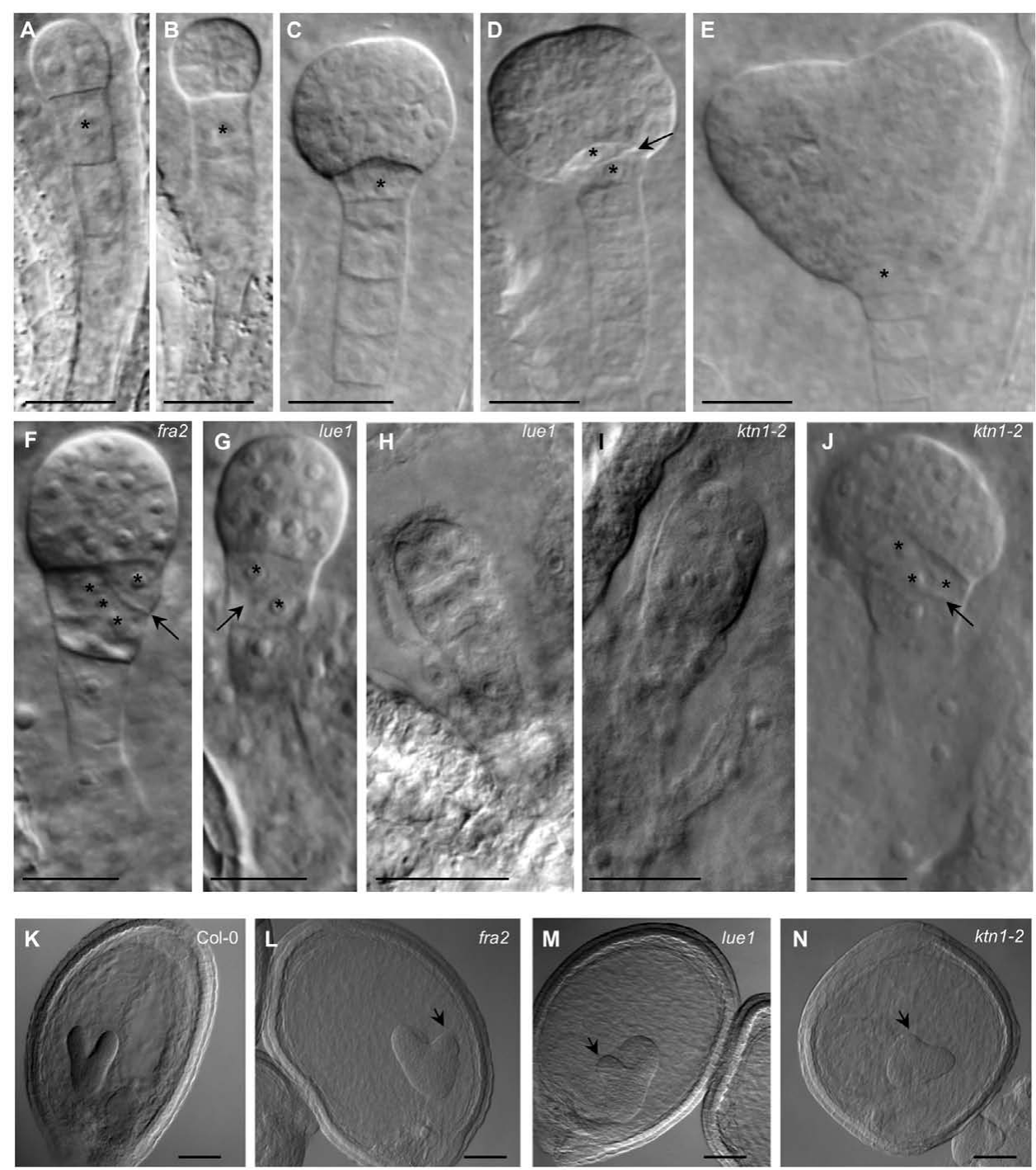

FIGURE 4 | Embryonic developmental defects in KATANIN 1 mutants. (A-E) Embryos of Col-0 at different developmental stages including 2 to 4 -cells (A), octant (B), early globular (C), transition (D), and heart shaped (E), showing typical organization of embryo proper and suspensor. Asterisks mark the position of the first suspensor cell $\mathbf{( A , B )}$ and the hypophysis in later stages (C-E). Transversal cell division of the hypophysis giving rise to quiescent center and columella stem cell is indicated by arrow (D). (F) Early globular embryo of the fra2 mutant showing slightly abnormal embryo proper and oblique cell division (arrow) at the hypophysis (asterisks). (G) Embryo of the lue1 mutant at early globular stage showing oblique cell division (arrow) at the hypophysis (asterisks). (H) Abnormaly elongated preglobular embryo of the lue1 mutant. (I) Abnormaly elongated preglobular embryo of the ktn1-2 mutant. (J) Globular embryo of the ktn1-2 mutant showing oblique cell division (arrow) at the hypophysis (asterisks). (K-N) The embryos at the heart shape stage of wild type Col-0 (K) and KATANIN 1 mutants fra2 (L), lue1 (M) and ktn1-2 (N). Note the asymmetry of the globular embryos in KATANIN 1 mutants (arrows). Scale bars $=20 \mu \mathrm{m}$.

flower, however, the overall picture of developed flowers was generally compromised. To gain insight into the low fertility defects in depth analysis of female and male reproductive tissues was conducted. Thorough phenotypic characterization of unpollinated ovule formation in KATANIN 1 mutants compared to Col-0 (Figures 2E-Q) revealed that ovule development was variably defective in KATANIN 1 mutants, ranging from severely malformed to normal and fertile. In detail female reproductive tissue analysis showed the following: $32 \%$ of the unfertilized ovules in fra2 showed a single secondary nucleus in their central cells and $14.4 \%$ showed unfused nuclei
(Figures $2 \mathrm{H}-\mathrm{J}, \mathbf{Q}$ ), in lue1 ovules, $33.6 \%$ contained a single secondary nucleus in their central cells and $4 \%$ contained unfused nuclei (Figures $\mathbf{2 K}-\mathbf{M}, \mathbf{Q}$ ), while in $k t n 1-2$ the percentages were 22.97 and $9.45 \%$, respectively (Figures $\mathbf{2 N - P , Q}$ ). Wild type plants showed very low occurence of such malformed ovules, 1.5 and $1.8 \%$, respectively (Figure 2Q). Together, these observations indicate problems with migration and the precise positioning of nuclei and probably in the fusion of the polar nuclei. Such malfunctions can create problems in the attraction and guidance of the sperm cells within the embryo sac. Moreover, analysis of KATANIN 1 mutant ovules also revealed deformed 

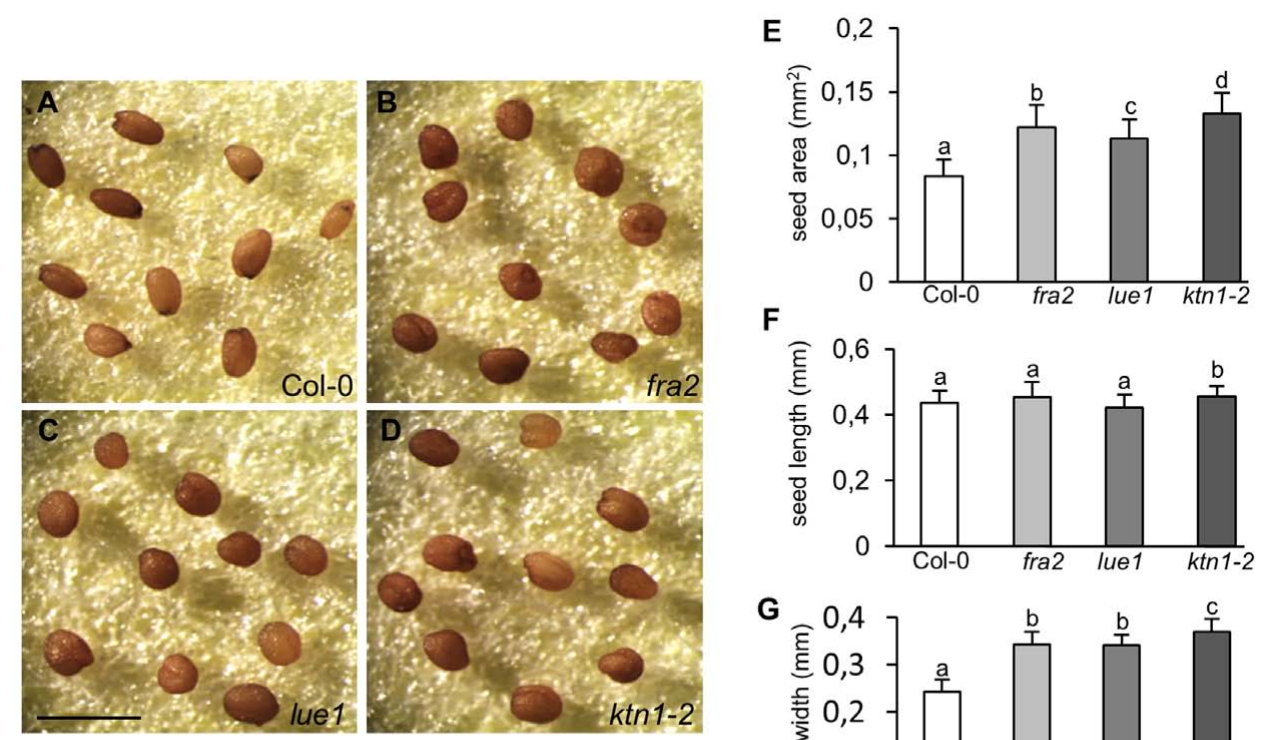

$\mathbf{F}$
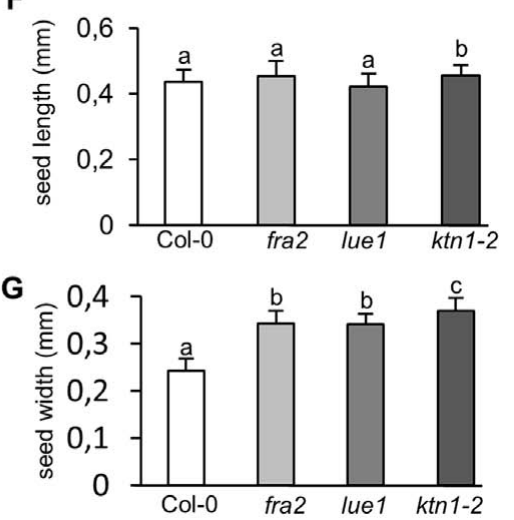

FIGURE 5 | Abnormal seed development in KATANIN 1 mutants. (A-D) Representative images of mature seeds in Col-0 and KATANIN 1 mutants. (A) Seeds of Col-0 exhibited a smooth contour and an elongated shape. (B-D) Seeds of fra2 (B), lue1 (C), and ktn1-2 (D) appeared more rounded and anomalous. (E) Seed area in Col-0 and KATANIN 1 mutants. (F) Seed length in Col-0 and KATANIN 1 mutants. (G) Seed width in Col-0 and KATANIN 1 mutants. Final calculations were based on data collection from 37 to 57 seeds. Different lowercase letters indicate statistical significance between treatments $(p<0.05)$. Error bars show \pm SD. Scale bar $=1 \mathrm{~mm}$.

inner and outer integuments in fra2, lue1, and ktn1-2 mutants suggesting sporophytic defects in the development of embryo sac (Supplemental Figures 2A-D).

\section{Anther Development in KATANIN 1 Mutants}

Since KATANIN p80 is essential for male fertility and sperm production in mouse (O'Donnell et al., 2012), we checked the formation of viable pollen in mature anthers of fra2, lue 1 and ktn1-2 mutants. Developed mature anther on the short stamens with stunt wider filaments of KATANIN 1 mutants, displayed also abnormal growth, as anther lobes were non-symmetrically and irregularly developed (Figures 3A-E). Alexander staining assays showed variable pollen viability in mature anther lobes of fra2, lue 1 and ktn1-2 as compared to the wild type, while degenerated inviable pollen could be also observed (Figures 3B,D,E).

\section{Aberrant Embryo Development in KATANIN 1 Mutants}

Embryonic development in KATANIN 1 mutants and in Col0 was examined with DIC optics. In Col-0 it was possible to discern several stages of embryo development which progressed orderly (ten Hove et al., 2015; Figures 4A-E). In all KATANIN 1 mutants we documented abnormalities in the embryo development, like misoriented cell divisions in the proembryo and the hypophysis (Figures $4 \mathbf{F}-\mathbf{J}$ ) and abnormally shaped hypophysis (Figures $4 \mathbf{F}, \mathbf{G}, \mathbf{J}$ ). In severe cases, particularly in the ktn1-2 mutant (Figures 4I,J), the shape of the embryo proper significantly deviated from its typical spherical form (Figure 4C). The redundant cell divisions in the ealry stages of embryogenenesis lead to malformed embryos of later developmental stages like heart shape stage (Figures $4 \mathrm{~L}-\mathrm{N}$ ) compared to Col-0 (Figure 4K).

\section{Abberant Seed Formation in KATANIN 1 Mutants}

Consistently with the previously described defects, the development of viable seeds is considerably compromised in KATANIN 1 mutants compared to Col-0. Mature seeds of fra2, lue1 and ktn1-2 were misshaped compared to Col-0 (Figures 5A-D), showing rounding and anomalous contour. Seed size analysis showed that KATANIN 1 mutants produce larger seeds compared to wild type. Quantification of the seed size parameters showed that all KATANIN 1 mutants display significantly larger seed areas (Figures 5A-E and Supplementary Table S1). In comparison to Col-0, seed length and width are differentially affected in KATANIN 1 mutants with the seed width to exhibit the most striking increase (Figures 5A-E). Differences in seed form parameters could be also observed among fra2, lue1, and $k t n 1-2$ (Figures 5E-G).

\section{Genetic Complementation and Rescue of Mutant Phenotypes}

To test whether GFP-KTN1 fusion protein can complement described phenotypes of KATANIN 1 mutants we transformed fra2, lue1 and ktn1-2 plants with pKTN1::GFP:KTN1 construct. 


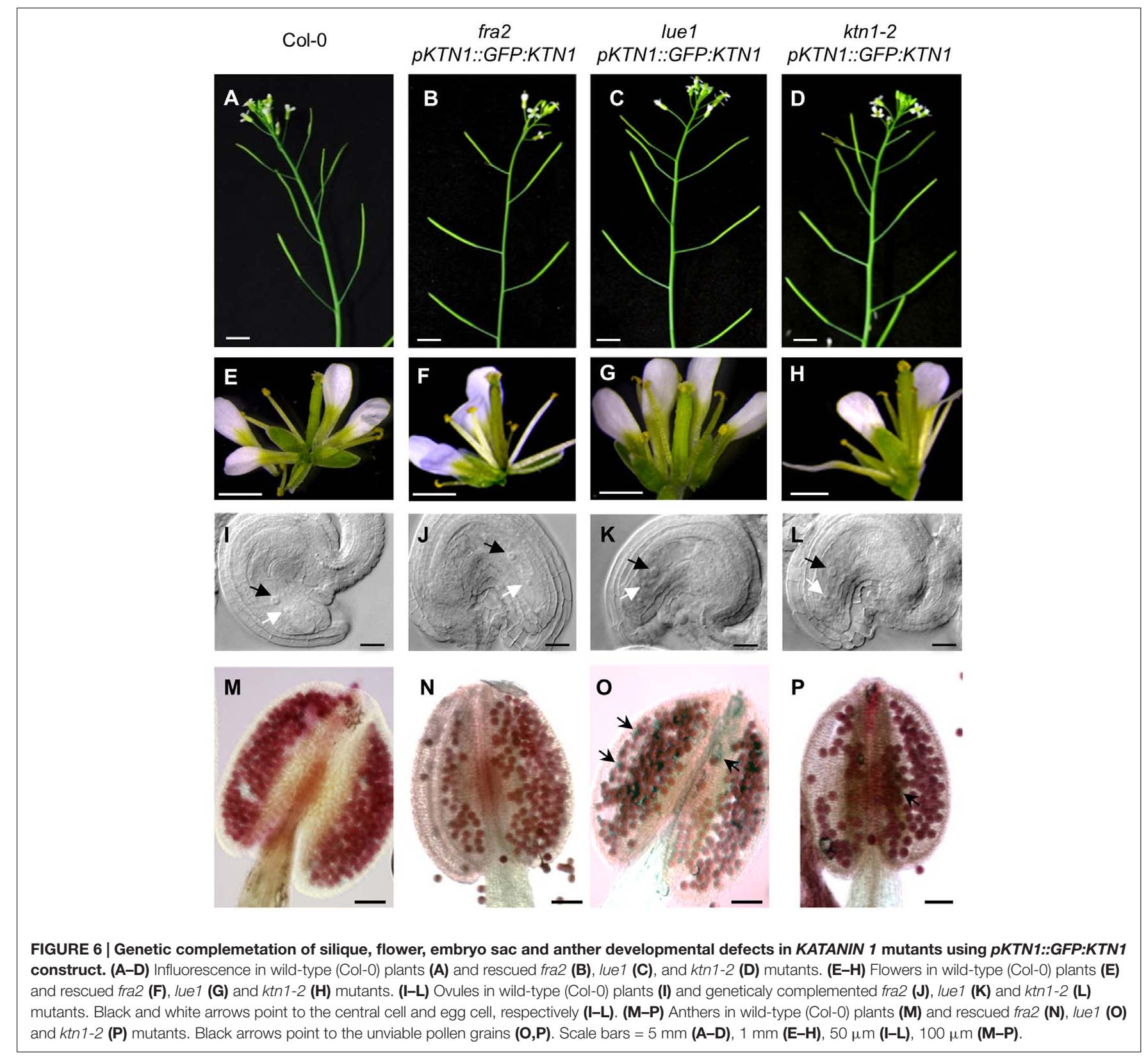

We analyzed T2 generation of transformed fra2, lue 1 and $k$ tn 1-2 mutants carrying $p K T N 1:: G F P: K T N 1$ constructs and we selected for further phenotypic analyses plants that harbor a single T-DNA copy $(n>200$, Chi-squared test $p<0.05)$. The phenotypes of fra2, lue 1 and ktn1-2 mutants could be rescued by introducing pKTN1::GFP:KTN1 construct. In detail, adult plants showed a wild type inflorescence architecture (Figures 6A-D) and flower development (Figures 6E-H). Concerning development of reproductive organs, introduction of $p K T N 1:: G F P: K T N 1$ rescued malformed phenotypes of ovules and anthers in KATANIN 1 mutants described above (Figures 6I-P). Nevertheless, in lue1 and ktn1-2 genetically complemented plants harboring pKTN1::GFP:KTN1 one can still detect some inviable pollen by Alexander staining, though in very low levels (Figures 6O,P).
Next, we checked development of seeds in such complemented fra2, lue 1 and ktn1-2 mutants and we observed rescue of embryo development (Figures 7A-H). Additionally, the complemented KATANIN 1 mutants displayed increased levels of fertilized seeds comparable to the wild type (Figures 7I-M) with minor differences among the mutants (Figure 7M).

Analysis of KATANIN 1 expression profile in the developing embryos of wild type (Col-0) transformed with pKTN1::GFP:KTN1 showed that it is expressed in all embryonic cells during the globular stage (Figure $\mathbf{8 A}$ ), at the triangle stage there is induction of KATANIN 1 expression at the embryonic root (Figure 8B) and in later developmental stages (heart to mature embryos) we could detect polarized KATANIN 1 expression in cotyledon poles and embryonic 


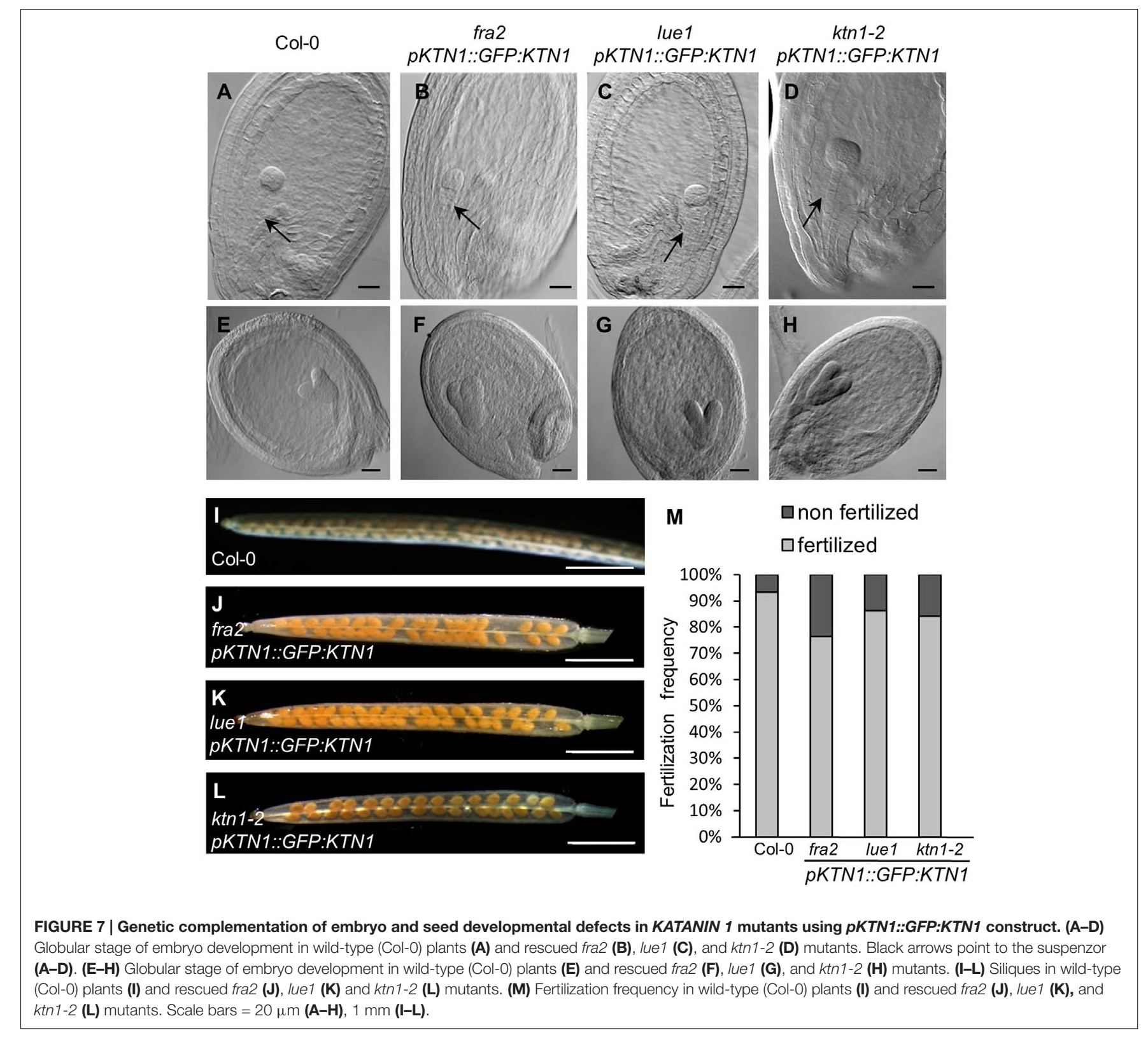

root (Figures $\mathbf{8 C}-\mathbf{G}$ ). These findings can help to explain the aberrant phenotypes of developing embryos in KATANIN 1 mutants. KATANIN 1 expression pattern analysis in genetically complemented mutants showed that in ktn 1-2 (Figures $\mathbf{8 V}-\mathbf{Z}$ ) it was consistent with polar patterning in wild type (Figures $8 \mathbf{A - G}$ ). In the case of genetically complemented fra 2 and lue 1 KATANIN 1 expression differed as it was generally distributed in most parts of the embryo while it was more prominent in the shoot apical meristem but almost not detectable in the embryonic root tip (Figures $\mathbf{8 H}-\mathbf{U}$ ). Western blot analysis for the detetion of GFP-KTN1 chimeric protein in the complemented fra2, lue1 and $k t n 1-2$ verified the presence of full-length GFP-KTN1 fusion protein only in lue1 genetic background, while in fra2, ktn1-2 and Col-0 we were able to detect only truncated forms of the GFP-KTN1 protein (in the case of fra2) or likely GFP alone driven by KATANIN 1 promoter (Figure 9). These findings suggest some complex unknown mechanisms involved in the regulation of expression pattern and distribution of KATANIN 1 protein in such plant lines.

\section{DISCUSSION}

Although the role of KATANIN 1 in the male and female gametogenesis is emerging in animal systems (O'Donnell et al., 2012; Joly et al., 2016; Tang et al., 2016) such function has not yet been found in plants. Future detailed genetic studies might eventually shed more light on gametophytic defects in the KATANIN 1 mutants. In this study, however, we focus on sporophytic developmental phenotypes of KATANIN 1 mutants 

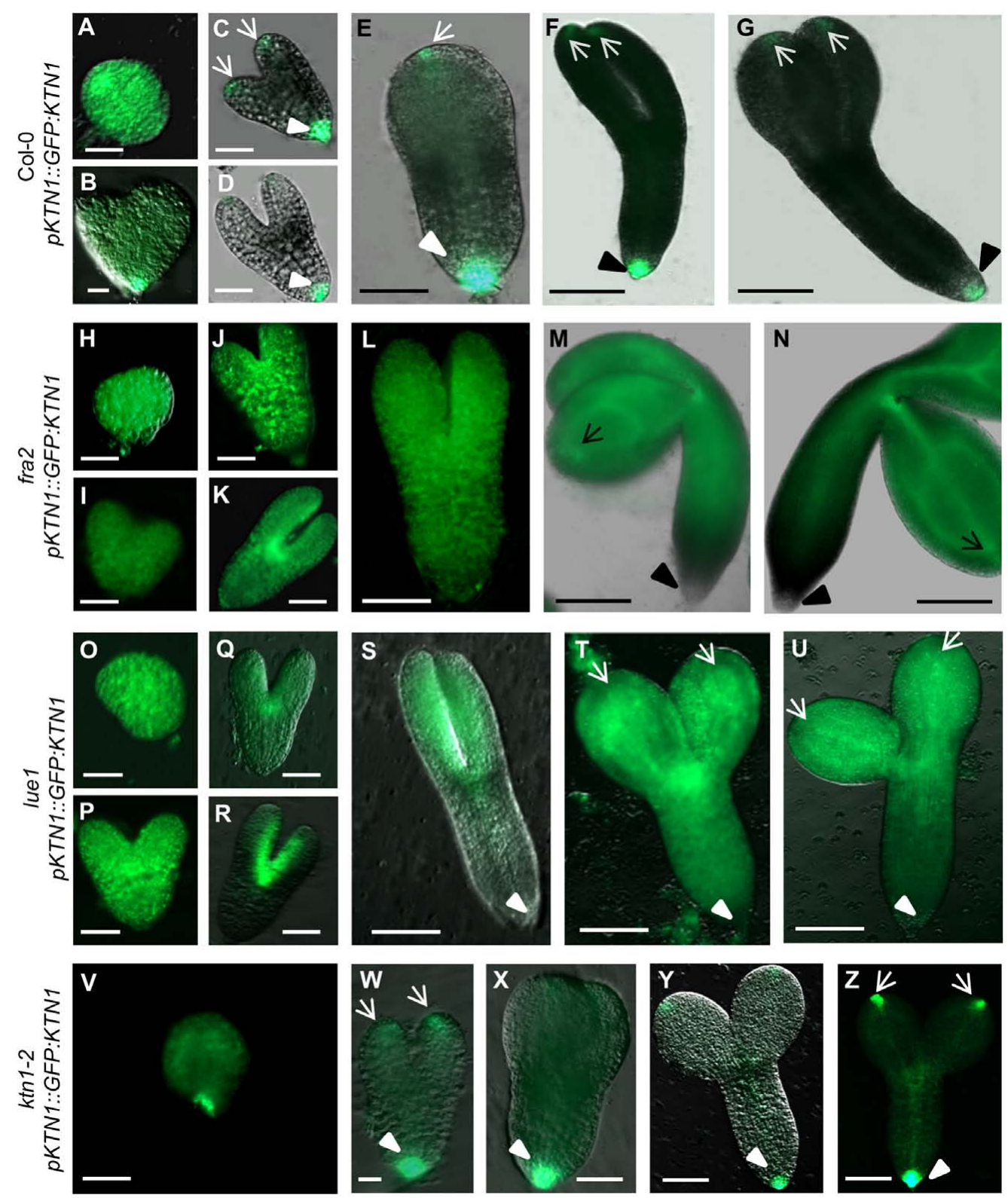

FIGURE 8 | KATANIN 1 expression in embryos in wild type and genetically complemented KATANIN 1 mutants. (A-F) different embryo developmental stages in wild-type (Col-0) plants expressing pKTN1::GFP:KTN1 construct: globular stage (A), triangle stage (B), heart stage (C), early torpedo (D), late torpedo (E), premature (F) and mature embryos (G). (H-N) different embryo developmental stages in rescued fra2 mutant plants expressing pKTN1::GFP:KTN1 construct: globular stage $\mathbf{( H )}$, triangle stage (I), heart stage $\mathbf{( J )}$, early torpedo $\mathbf{( K )}$, late torpedo $\mathbf{( L )}$, premature $\mathbf{( M )}$, and mature embryos (N). (O-U) GFP-KTN1 fusion protein localization in different embryo developmental stages in rescued lue1 mutant plants expressing pKTN1::GFP:KTN1 construct: globular stage (O), triangle stage (P), heart stage (Q), early torpedo (R), late torpedo (S), premature (T), and mature embryos (U). (V-Z) different embryo developmental stages in rescued ktn1-2 mutant plants expressing pKTN1::GFP:KTN1 construct: globular stage $(\mathbf{V})$, heart stage $(\mathbf{W})$, torpedo $(\mathbf{X})$, premature $(\mathbf{Y})$, and mature embryos $(\mathbf{Z})$. Arrows indicate cotyledon poles, arrow-heads indicate embryonic root meristem (A-Z). Scale bars $=20 \mu \mathrm{m}(\mathbf{A}-\mathbf{D}, \mathbf{H}-\mathbf{K}, \mathbf{O}-\mathbf{R}, \mathbf{V}-\mathbf{W})$ and $100 \mu \mathrm{m}$ (E-G, L-N, S-U, X-Z).

during embryogenesis and seed formation including a thorough description of developmental defects which are genetically rescued by appropriate $p K T N 1:: G F P: K T N 1$ functional construct.

\section{KATANIN 1 Role in Embryo Sac Polarity}

The regulation of microtubule organization and dynamics is critical for many cellular processes during plant development.
Gametogenesis is the process by which gametes undergo mitotic and meiotic division and differentiation to produce germ units capable of motility and fertilization. Studies have shown that the microtubule-based cytoskeleton that provides the track for the transport of spermatids and cell organelles during spermatogenesis is likely working in concert with the actin cytoskeleton (Tang et al., 2013, 2016; O'Donnell and O'Bryan, 


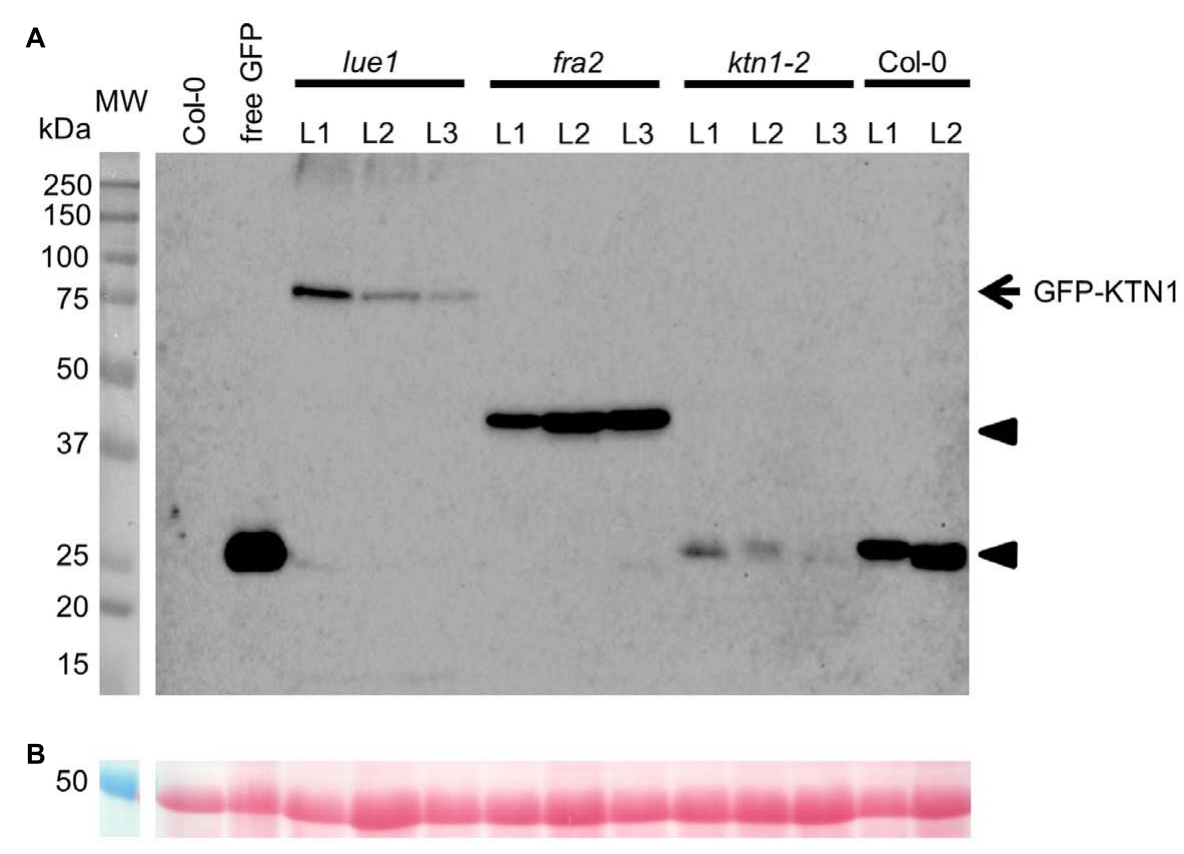

FIGURE 9 | Western blot detection of GFP-KTN1 in genetically complemented fra2, lue1, and ktn1-2 mutants carrying pKTN1::GFP:KTN1 construct. (A) GFP-KTN1 protein (85 kDa) confirmed by using anti-GFP antibody (arrow) shows the chimeric GFP-KTN1 protein in /ue1 genetic background while arrow heads show truncated and cleaved forms of GFP-KTN1 in fra2, ktn1-2 and Col-0. (B) Loading control (proteins stained by Ponceau).

2014). The microtubule cytoskeleton is essential for polar organelle distribution including arrangement and migration of nuclei in developing embryo sac (Russell, 1993; Webb and Gunning, 1994). Moreover, radial perinuclear microtubules support cellularization that leads to the formation of the sevencelled embryo sac (Webb and Gunning, 1994). Extensive arrays of microtubules are present throughout the megagametogenesis and can be categorized into the cytoplasmic microtubules and the nuclear-associated networks typical for egg cell and two- and four-nucleate stages (Webb and Gunning, 1994). They possibly regulate the migration and precise positioning of nuclei in these multinucleate cells and the sperm cell guidance within the degenerated synergid (Webb and Gunning, 1994). So, based on cytological observations the defects detected in KATANIN 1 mutant embryo sacs can be attributed to the lack of rapid microtubule rearranging ability due to the missing microtubule severing activity.

Moreover, we show here that these embryo sac polarity defects were resued by $p$ KTN1::GFP:KTN1 construct.

\section{KATANIN 1 Role in Embryo and Seed Development}

Cytoskeletal rearrangements and dynamic changes have been rarely studied during diverse stages of plant fertilization and embryo development. Fertilization triggers the completion of meiosis while short acentrosomal meiotic spindle must be disassembled and right afterward replaced by big mitotic spindle filling most of the zygote volume (Albertson, 1984; Kemphues et al., 1986; Albertson and Thomson, 1993; McCarter et al., 1999;
Yang et al., 2003). This quick microtubule remodeling has to be under precise developmental control.

In animal systems recent studies have shed light into the role of katanin severing complex during the female meiotic spindle assemby (Joly et al., 2016) in Caenorhabditis elegans and during sperm production in mouse (O'Donnell et al., 2012). Additionally, katanin complex severing microtubules is under tight regulation during the transition form the meiotic to mitotic stage to allow proper embryogenesis, as its persistence could have detrimental effects (Beard et al., 2016). Previous studies in Arabidopsis roots showed that fra 2 and lue 1 mutants show mitotic spindle multipolarity and rotation compared to Col-0, suggesting that aberrant spindle positioning in KATANIN 1 mutants may be the mechanistic basis for misorientation of the cell division plane (Panteris et al., 2011; Panteris and Adamakis, 2012). The strict pattern of cell divisions guiding development of wild-type embryos has been well previously described (Jürgens and Mayer, 1994). In this study we report that KATANIN 1 mutants show defects in cell division plane orientation not restricted to particular stages or cells of the developing embryos, resulting in malformation of embryos. Concomitantly seed development is also compromised in KATANIN 1 mutants.

Frequencies and patterns of cell divisions in embryo, endosperm and integuments dictate size of seeds. It has also been reported that ovule abortion or embryo lethality is correlated with the production of enlarged seeds (Sornay et al., 2016). Given the problems described in KATANIN 1 mutant with ovule abortion and embryo development the formation of enlarged seeds corroborates these results. 
Importantly, most of above-described abberant embryo and seed phenotypes in KATANIN 1 mutants were almost fully (in the case of lue 1 possessing full-length GFP-KTN1) or at least partialy (in the case of fra2 and $k t n 1-2$ ) genetically rescued by functional $p K T N 1:: G F P: K T N 1$ construct. Although GFP-KTN1 chimeric protein might be cleaved (in the case of ktn1-2) or truncated (in the case of fra2) it can still complement mutant phenotypes. Moreover, results from developing embryos might suggest that patterning and spatiotemporal distribution of GFP driven by KATANIN 1 promoter might differ from those of full-length or truncated KATANIN 1 proteins driven by the same promoter.

\section{AUTHOR CONTRIBUTIONS}

IL carried out most of the experiments conducted herein. GK helped with embryo preparations and with microscopy in general. JS conceived the project and supervised its progress. DS and JŠ wrote the manuscript with input by the other authors.

\section{REFERENCES}

Abu-Abied, M., Mordehaev, I., Sunil Kumar, G. B., Ophir, R., Wasteneys, G. O., and Sadot, E. (2015a). Analysis of microtubule-associated-proteins during IBA-mediated adventitious root induction reveals KATANIN dependent and independent alterations of expression patterns. PLOS ONE 10:e0143828. doi: 10.1371 /journal.pone. 0143828

Abu-Abied, M., Rogovoy Stelmakh, O., Mordehaev, I., Grumberg, M., Elbaum, R., Wasteneys, G. O., et al. (2015b). Dissecting the contribution of microtubule behaviour in adventitious root induction. J. Exp. Bot. 66, 2813-2824. doi: 10.1093/jxb/erv097

Albertson, D. G. (1984). Formation of the first cleavage spindle in nematode embryos. Dev. Biol. 101, 61-72. doi: 10.1016/0012-1606(84)90117-9

Albertson, D. G., and Thomson, J. N. (1993). Segregation of holocentric chromosomes at meiosis in the nematode, Caenorhabditis elegans. Chromosom. Res. 1, 15-26. doi: 10.1007/BF00710603

Beard, S. M., Smitk, R. B., Chan, B. G., and Mains, P. E. (2016). Regulation of the MEI-1/MEI-2 microtubule-severing Katanin complex in early Caenorhabditis elegans development. G3 6, 3257-3268. doi: 10.1534/g3.116. 031666

Bichet, A., Desnos, T., Turner, S., Grandjean, O., and Höfte, H. (2001). BOTERO1 is required for normal orientation of cortical microtubules and anisotropic cell expansion in Arabidopsis. Plant J. 25, 137-148. doi: 10.1111/j.1365-313X.2001. 00946.x

Bouquin, T., Mattsson, O., Naested, H., Foster, R., and Mundy, J. (2003). The Arabidopsis lue1 mutant defines a katanin p60 ortholog involved in hormonal control of microtubule orientation during cell growth. J. Cell Sci. 116, 791-801. doi: $10.1242 /$ jcs. 00274

Burk, D. H., Liu, B., Zhong, R., Morrison, W. H., and Ye, Z. H. (2001). A kataninlike protein regulates normal cell wall biosynthesis and cell elongation. Plant Cell 13, 807-827. doi: 10.1105/tpc.13.4.807

Burk, D. H., and Ye, Z. H. (2002). Alteration of oriented deposition of cellulose microfibrils by mutation of a katanin-like microtubule-severing protein. Plant Cell 14, 2145-2160. doi: 10.1105/tpc.003947

Hartman, J. J., Mahr, J., McNally, K., Okawa, K., Iwamatsu, A., Thomas, S., et al. (1998). Katanin, a microtubule-severing protein, is a novel AAA ATPase that targets to the centrosome using a WD40-containing subunit. Cell 93, 277-287. doi: 10.1016/S0092-8674(00)81578-0

Hartman, J. J., and Vale, R. D. (1999). Microtubule disassembly by ATPdependent oligomerization of the AAA enzyme katanin. Science 286, 782-785. doi: $10.1126 /$ science. 286.5440 .782

\section{FUNDING}

This work was funded by Czech Science Foundation GAČR, project Nr. 15-19284 and Endowment Fund of the Palacky University in Olomouc.

\section{ACKNOWLEDGMENTS}

We thank Drs. Ioannis Adamakis and Emanuel Panteris and Masayoshi Nakamura for kindly providing published materials. We thank Markéta Trajerová for technical help and Dr. Anna Kuchařová for primer design.

\section{SUPPLEMENTARY MATERIAL}

The Supplementary Material for this article can be found online at: http://journal.frontiersin.org/article/10.3389/fpls.2017.00728/ full\#supplementary-material

Joly, N., Martino, L., Gigant, E., Dumont, J., and Pintard, L. (2016). Microtubulesevering activity of AAA-ATPase Katanin is essential for female meiotic spindle assembly. Development 143, 3604-3614. doi: 10.1242/dev.140830

Jürgens, G., and Mayer, U. (1994). "Arabidopsis," in A Colour Atlas of Developing Embryos, ed. J. Bard (London: Wolfe Publishing), 7-21.

Keech, O., Pesquet, E., Gutierrez, L., Ahad, A., Bellini, C., Smith, S. M., et al. (2010). Leaf senescence is accompanied by an early disruption of the microtubule network in Arabidopsis. Plant Physiol. 154, 1710-1720. doi: 10.1104/pp.110. 163402

Kemphues, K. J., Wolf, N., Wood, W. B., and Hirsh, D. (1986). Two loci required for cytoplasmic organization in early embryos of Caenorhabditis elegans. Dev. Biol. 113, 449-460. doi: 10.1016/0012-1606(86)90180-6

Lindeboom, J. J., Nakamura, M., Hibbel, A., Shundyak, K., Gutierrez, R., Ketelaar, T., et al. (2013). A mechanism for reorientation of cortical microtubule arrays driven by microtubule severing. Science 342:e1245533. doi: 10.1126/ science. 1245533

McCarter, J., Bartlett, B., Dang, T., and Schedl, T. (1999). On the control of oocyte meiotic maturation and ovulation in Caenorhabditis elegans. Dev. Biol. 205, 111-128. doi: 10.1006/dbio.1998.9109

McClinton, R. S., Chandler, J. S., and Callis, J. (2001). cDNA isolation, characterization, and protein intracellular localization of a katanin-like p60 subunit from Arabidopsis thaliana. Protoplasma 216, 181-190. doi: 10.1007/ BF02673870

McNally, F. J., and Vale, R. D. (1993). Identification of katanin, an ATPase that severs and disassembles stable microtubules. Cell 75, 419-429. doi: 10.1016/ 0092-8674(93)90377-3

Mukherjee, S., Diaz Valencia, J. D., Stewman, S., Metz, J., Monnier, S., Rath, U., et al. (2012). Human Fidgetin is a microtubule severing the enzyme and minusend depolymerase that regulates mitosis. Cell Cycle 11, 2359-2366. doi: 10.4161/ cc. 20849

Nakamura, M. (2015). Microtubule nucleating and severing enzymes for modifying microtubule array organization and cell morphogenesis in response to environmental cues. New Phytol. 205, 1022-1027. doi: 10.1111/nph.12932

Nakamura, M., Ehrhardt, D. W., and Hashimoto, T. (2010). Microtubule and katanin-dependent dynamics of microtubule nucleation complexes in the acentrosomal Arabidopsis cortical array. Nat. Cell Biol. 12, 1064-1070. doi: $10.1038 /$ ncb2110

O’Donnell, L., and O’Bryan, M. K. (2014). Microtubules and spermatogenesis. Semin. Cell Dev. Biol. 30, 45-54. doi: 10.1016/j.semcdb.2014.01.003

O’Donnell, L., Rhodes, D., Smith, S. J., Merriner, D. J., Clark, B. J., Borg, C., et al. (2012). An essential role for katanin p80 and microtubule severing in 
male gamete production. PLoS Genet. 8:e1002698. doi: 10.1371/journal.pgen. 1002698

Panteris, E., and Adamakis, I. D. (2012). Aberrant microtubule organization in dividing root cells of p60-katanin mutants. Plant Signal. Behav. 7, 16-18. doi: $10.4161 /$ psb.7.1.18358

Panteris, E., Adamakis, I. D., Voulgari, G., and Papadopoulou, G. (2011). A role for katanin in plant cell division: microtubule organization in dividing root cells of fra2 and lue1 Arabidopsis thaliana mutants. Cytoskeleton 68, 401-413. doi: $10.1002 / \mathrm{cm} .20522$

Qu, J., Ye, J., Geng, Y. F., Sun, Y. W., Gao, S. Q., Zhang, B. P., et al. (2012). Dissecting functions of KATANIN and WRINKLED1 in cotton fiber development by virus-induced gene silencing. Plant Physiol. 160, 738-748. doi: $10.1104 /$ pp.112.198564

Roll-Mecak, A., and Vale, R. D. (2005). The Drosophila homologue of the hereditary spastic paraplegia protein, spastin, severs and disassembles microtubules. Curr. Biol. 15, 650-655. doi: 10.1016/j.cub.2005.02.029

Russell, S. D. (1993). The egg cell: development and role in fertilization and early embryogenesis. Plant Cell 5, 1349-1359. doi: 10.1105/tpc.5.10.1349

Soga, K., Yamaguchi, A., Kotake, T., Wakabayashi, K., and Hoson, T. (2010). 1Aminocyclopropane-1-carboxylic acid ACC-induced reorientation of cortical microtubules is accompanied by a transient increase in the transcript levels of gamma-tubulin complex and katanin genes in azuki bean epicotyls. J. Plant Physiol. 167, 1165-1171. doi: 10.1016/j.jplph.2010.04.001

Sornay, E., Dewitte, W., and Murray, J. A. H. (2016). Seed size plasticity in response to embryonic lethality conferred by ectopic CYCD activation is dependent on plant architecture. Plant Signal. Behav. 11:e1192741. doi: 10.1080/15592324. 2016.1192741

Stoppin-Mellet, V., Gaillard, J., Timmers, T., Neumann, E., Conway, J., and Vantard, M. (2007). Arabidopsis katanin binds microtubules using a multimeric microtubule-binding domain. Plant Physiol. Biochem. 45, 867-877. doi: 10.1016/j.plaphy.2007.09.005

Stoppin-Mellet, V., Gaillard, J., and Vantard, M. (2006). Katanin's severing activity favors bundling of cortical microtubules in plants. Plant J. 46, 1009-1017. doi: 10.1111/j.1365-313X.2006.02761.x

Tang, E. I., Lee, W. M., and Cheng, C. (2016). Coordination of actin- and microtubule-based cytoskeletons support transport of spermatids and residual bodies/phagosomes during spermatogenesis in the testis of the male rat. Endocrinology 157, 1644-1659. doi: 10.1210/en.2015-1962
Tang, E. I., Mruk, D. D., and Cheng, C. Y. (2013). MAP/microtubule affinityregulating kinases, microtubule dynamics, and spermatogenesis. J. Endocrinol. 217, R13-R23. doi: 10.1530/JOE-12-0586

ten Hove, C. A., Lu, K. J., and Weijers, D. (2015). Building a plant: cell fate specification in the early Arabidopsis embryo. Development 142, 420-430. doi: $10.1242 / \mathrm{dev} .111500$

Webb, M., Jouannic, S., Foreman, J., Linstead, P., and Dolan, L. (2002). Cell specification in the Arabidopsis root epidermis requires the activity of ECTOPIC ROOT HAIR 3-a katanin-p60 protein. Development 129, 123-131.

Webb, M. C., and Gunning, B. E. S. (1994). Embryo sac development in Arabidopsis. II. The cytoskeleton during megagametogenesis. Sex. Plant Reprod. 7, 153-163. doi: 10.1007/BF00228488

Wightman, R., Chomicki, G., Kumar, M., Carr, P., and Turner, S. R. (2013). SPIRAL2 determines plant microtubule organization by modulating microtubule severing. Curr. Biol. 23, 1902-1907. doi: 10.1016/j.cub.2013. 07.061

Wightman, R., and Turner, S. R. (2007). Severing at sites of microtubule crossover contributes to microtubule alignment in cortical arrays. Plant J. 52, 742-751. doi: 10.1111/j.1365-313X.2007.03271.x

Yang, C.-Y., Spielman, M., Coles, J. P., Li, Y., Ghelani, S., Bourdon, V., et al. (2003). TETRASPORE encodes a kinesin required for male meiotic cytokinesis in Arabidopsis. Plant J. 34, 229-240. doi: 10.1046/j.1365-313X.2003. 01713.x

Zhang, Q., Fishel, E., Bertroche, T., and Dixit, R. (2013). Microtubule severing at crossover sites by katanin generates ordered cortical microtubule arrays in Arabidopsis. Curr. Biol. 23, 2191-2195. doi: 10.1016/j.cub.2013. 09.018

Conflict of Interest Statement: The authors declare that the research was conducted in the absence of any commercial or financial relationships that could be construed as a potential conflict of interest.

Copyright (c) 2017 Luptovčiak, Samakovli, Komis and Šamaj. This is an open-access article distributed under the terms of the Creative Commons Attribution License (CC BY). The use, distribution or reproduction in other forums is permitted, provided the original author(s) or licensor are credited and that the original publication in this journal is cited, in accordance with accepted academic practice. No use, distribution or reproduction is permitted which does not comply with these terms. 